\title{
A simplified lung ultrasound for the diagnosis of interstitial lung disease in connective tissue disease: a meta-analysis
}

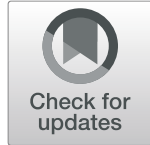

Hai Qin Xie, Wei Wei Zhang, De Sheng Sun, Xiang Mei Chen, Shu Fang Yuan, Zheng Hua Gong* (10 and Li Liu*

\begin{abstract}
Background: Interstitial lung disease (ILD) is a common complication of connective tissue disease (CTD) and a leading cause of morbidity and mortality. There are various lung ultrasound (LUS) scoring systems with different lung intercostal spaces (LIS). The purpose of this meta-analysis was to find a simplified LUS method for the assessment of CTD-ILD.
\end{abstract}

Methods: We systematically retrieved lung ultrasound diagnostic studies on CTD-ILD in PubMed, Embase, and Web of Science databases. Summary diagnostic accuracy, including sensitivity, specificity, and area under the curve (AUC), was analyzed. Subgroup analysis was conducted according to different LIS and diseases.

Results: The 11 studies included in this meta-analysis comprised a total of 487 patients with CTD. The pooled sensitivity and specificity of the LUS were 0.859 (95\% confidence interval (CI) 0.812-0.898) and 0.839 (95\% Cl 0.782-0.886),

respectively, illustrating its great value for CTD-ILD diagnosis. In addition, there were six methods to evaluate LIS, including 72, 65, 50, 14, 10, and all LIS. The pooled sensitivity and specificity of 14 LIS were 0.982 (95\% Cl 0.904-1.000) and 0.875 (95\% Cl 0.710-0.965), respectively. The pooled positive likelihood ratio (PLR), negative likelihood ratio (NLR), and diagnostic odd ratio (DOR) of 14 LIS were 7.297 (95\% Cl 6.050-17.45), 0.029 (95\% Cl 0.006-0.147), and 292.30 (95\% Cl 35.53-2403.8), respectively. Moreover, the AUC for systemic sclerosis (SSc) and rheumatoid arthritis (RA) was 0.929 and 0.981, respectively; the DOR for SSC and RA was 42.93 (95\% Cl 17.75-103.79) and 80.24 (95\% Cl 8.107-796.09), respectively.

Conclusions: We found a modified and simplified method of LUS, by scanning 14 LIS in a short time, which had a very high sensitivity and specificity.

Keywords: Lung ultrasound, Interstitial lung disease, Connective tissue disease, Meta-analysis

\section{Introduction}

Interstitial lung disease (ILD) is a common complication of connective tissue disease (CTD) and a leading cause of morbidity and mortality [1]. Thus, early diagnosis and treatment may improve the prognosis of patients with ILD [2].

High-resolution computed tomography (HRCT) is the gold standard for ILD diagnosis [3-5]. It can detect the location and type of lesions through its high resolution. Unfortunately, it is hampered by high cost and potential risks associated with radiation exposure, especially for pregnant women. Accordingly, finding a low-cost, non-invasive, and

\footnotetext{
* Correspondence: gongzhenghua2018@126.com; liuli126126@126.com Department of Ultrasound, Peking University Shenzhen Hospital, Lianhua Road 1120, FuTian District, Shenzhen 518036, Guangdong, China
}

non-ionizing diagnostic method is necessary for ILD. Lung ultrasound (LUS) has all of these advantages and is an accessible bedside procedure. As a result, it is easily accepted by patients. Over the last 20 years, LUS has mainly been applied in CTD-ILD diagnosis, where it has shown high sensitivity and specificity. The assessment of ILD by LUS is determined by the number of B-lines, which appear as a comet tail signal and originate from the pleural line without fading to the edge of the screen [6].

The total number of B-lines was found to correlate well with the HRCT score [7]. To assess the number of B-lines, previous studies used various scoring systems by designing different intercostal spaces (LIS), such as 72 LIS, 50 LIS, and 14. LIS [7-10]. In fact, 70 and 50 LIS

(C) The Author(s). 2019 Open Access This article is distributed under the terms of the Creative Commons Attribution 4.0 International License (http://creativecommons.org/licenses/by/4.0/), which permits unrestricted use, distribution, and 
were time-consuming and hard to perform daily. Up to now, there have been few data about which LIS should be better studied for calculating the number of B-lines by LUS. Accordingly, a meta-analysis is needed to find a simplified LUS method for CTD-ILD diagnosis.

\section{Methods}

\section{Search strategy and selection studies}

The PubMed, Embase, and Web of Science databases were searched up to October 31, 2018. Two investigators independently searched the databases and screened the articles. Disagreements were resolved by a third investigator. All studies found were in English. We used various combinations of Medical Subject Heading (MSH) terms, including ultrasound, sonography, lung, interstitial, pulmonary fibrosis, and connective tissue disease. The search string also included B-line and high-resolution computed tomography. Selected studies were about the LUS diagnostic value according to the B-lines in patients with CTD-ILD, compared to HRCT. All the references mentioned in the selected studies were reviewed to avoid omitting studies not indexed by the electronic databases. Articles with overlapping data or insufficient data, conference abstracts, reviews, and meta-analyses were excluded.

\section{Data extraction and quality assessment}

Data from the included studies were extracted independently by two researchers and consisted of the characteristic features, such as the author, publication year, country, patients' sex and mean age, number of LIS, cutoff values of the B-lines, probe frequency, probe type, mean disease duration, number of LUS operators, and kappa value. True positive, false positive, true negative, and false negative were obtained from the selected studies. LUS diagnosis of CTD-ILD was scored by the number of B-lines, using HRCT as the golden standard. The quality of each article was evaluated by means of Quality Assessment of Diagnostic Accuracy Studies (QUADAS) [11].

\section{Statistical analysis}

The heterogeneity was evaluated using the $I^{2}$ statistic. When the $I^{2}$ of heterogeneity was greater than 50, we used the random effect model. Otherwise, the fixed effect model [12] was used. Summary sensitivity, specificity, positive likelihood ratio (PLR), negative likelihood ratio (NLR), and diagnostic odds ratio (DOR) were measured. The $95 \%$ confidence interval (CI) was calculated for individual and pooled data. In addition, we analyzed the summary receiver-operating characteristic curves (SROC), area under the curve (AUC), and $Q$ index for all studies. The subgroup analysis was performed according to the number of LIS and different diseases. The software used was Meta-DiSc (version 1.4, Ramon y Cajal Hospital, Madrid, Spain) [13].

\section{Results}

Literature inclusion and data extraction

We retrieved 110 articles in the primary search. After reading the titles and abstracts, 29 articles were selected for reviewing the full text. Two studies were omitted because they were review or meta-analysis. Seven studies were omitted due to the qualitative diagnosis. Three studies were omitted because they mainly compared two LUS methods. The results indicated a highly significant correlation between two methods without the data about the diagnostic accuracy [14-16]. Three studies were deleted because they described a good correlation between B-line score of LUS and the Warrick score on HRCT, but no sensitivity and specificity data were provided [17-19]. Three studies were excluded because they mainly focused on the diagnosis of ILD by LUS with the sign of pleural irregularity, not with the number of B-lines [20-22]. Ultimately, 11 articles were included, comprising a total of 487 patients (Fig. 1). We extracted the data from the 11 articles (Tables 1 and 2) and measured the LUS diagnostic value in CTD-ILD. One study analyzed three diseases, including systemic lupus erythematosus (SLE), rheumatoid arthritis (RA), and systemic sclerosis ( $\mathrm{SSc}$ ), and separately calculated the diagnostic accuracy of each [23]. Ten of the 11 studies acquired the scores of QUADAS more than 10 (Table 3).

\section{Diagnostic accuracy of LUS in different LIS}

A total of 11 articles were pooled together to calculate the summary diagnostic accuracy of LUS in patients with CTD-ILD (Table 4 and Fig. 2). The sum of patients with ILD was 266 and 221 without ILD. The pooled sensitivity and specificity of LUS were 0.859 (95\% CI $0.812-$ 0.898 ) and 0.839 (95\% CI 0.782-0.886), respectively. In general, the pooled PLR, NLR, and DOR were 5.412 (95\% CI 3.026-9.680), 0.176 (95\% CI 0.111-0.279), and

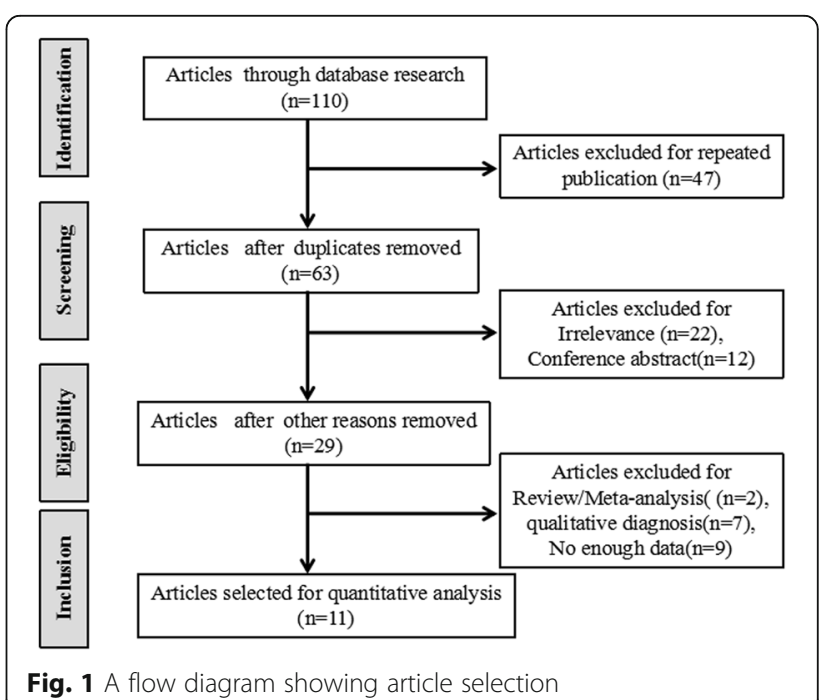




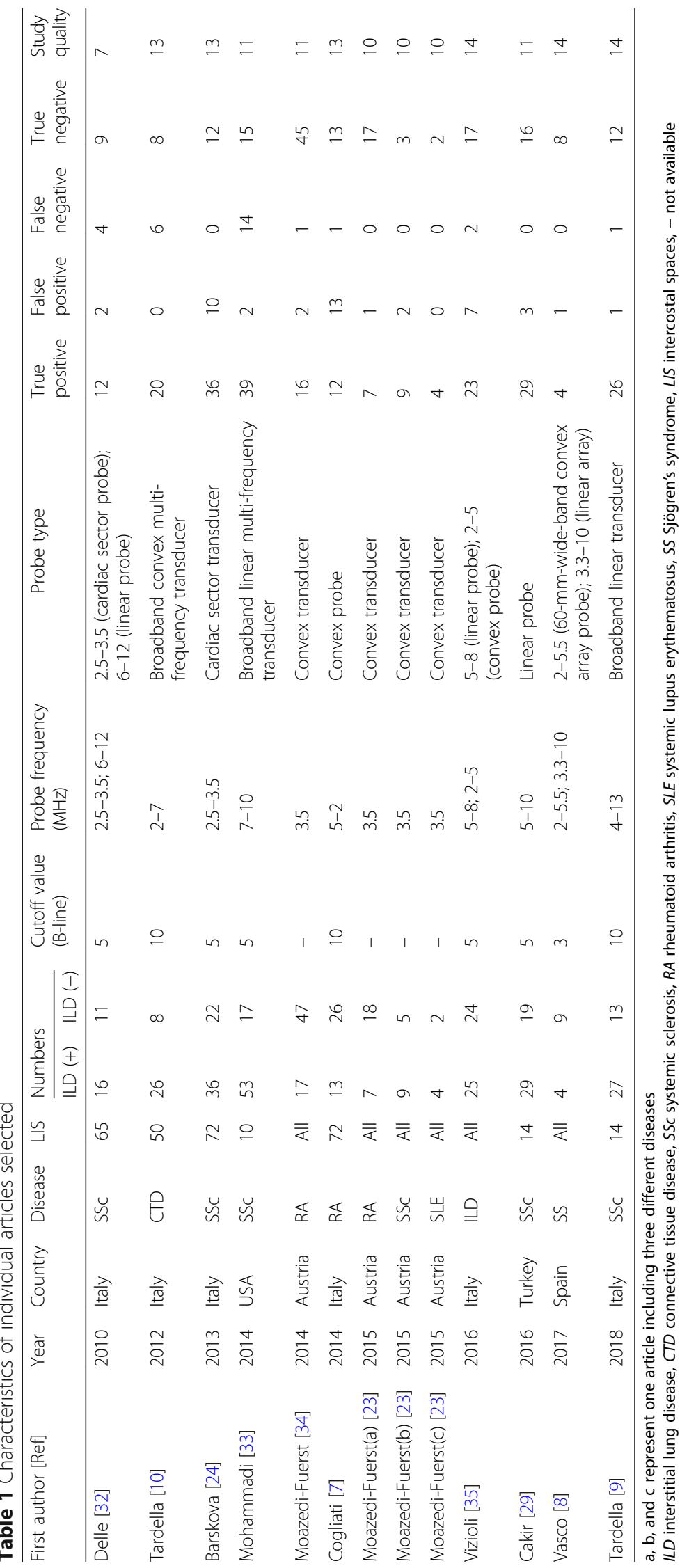


Table 2 Demographic characteristics of study populations

\begin{tabular}{|c|c|c|c|c|c|c|c|}
\hline \multirow[t]{2}{*}{ First author [Ref] } & \multicolumn{2}{|c|}{ Numbers } & \multirow{2}{*}{$\begin{array}{l}\text { Mean age (range) } \\
\text { years } \pm \text { standard } \\
\text { deviation }\end{array}$} & \multirow{2}{*}{$\begin{array}{l}\text { Mean disease duration } \\
\text { (months, years) }\end{array}$} & \multirow{2}{*}{$\begin{array}{l}\text { Spend time } \\
\text { (min) }\end{array}$} & \multirow{2}{*}{$\begin{array}{l}\text { Number of } \\
\text { LUS operator }\end{array}$} & \multirow{2}{*}{$\begin{array}{l}\text { Reproducibility between } \\
\text { operator }\end{array}$} \\
\hline & Female & Male & & & & & \\
\hline Delle [32] & 22 & 3 & $53 \pm 10.5$ & - & - & 2 & Intra-class correlation $=0.681$ \\
\hline Tardella [10] & 30 & 4 & $57 \pm 13$ & $85.58 \pm 84.37$ months & $23 \pm 4.5$ & 2 & Kappa $=0.846-0.980$ \\
\hline Barskova [24] & 54 & 4 & $51 \pm 14$ & - & $<10$ & 2 & $\begin{array}{l}\text { Intra-observer variability } 5.1 \% \text {, } \\
\text { inter-observer variability } 7.4 \%\end{array}$ \\
\hline Mohammadi [33] & 62 & 8 & $50.29 \pm 9.7$ & $88 \pm 83.1$ months & - & 1 & Kappa $=0.838$ \\
\hline Moazedi-Fuerst [34] & 54 & 10 & $59 \pm 12$ & $9.4(2-21)$ years & - & 2 & Kappa $=0.92$ \\
\hline Cogliati [7] & 29 & 10 & 64.87 & 11.21 years & - & 2 & Kappa $=0.78$ \\
\hline Moazedi-Fuerst [23] & 38 & 7 & $54(28-74)$ & $8(1-35)$ years & - & 2 & - \\
\hline Vizioli [35] & - & - & $65 \pm 13$ & & $8 \pm 1(5-14)$ & & - \\
\hline Cakir [29] & 46 & 2 & $50.8 \pm 11.9$ & $4.6 \pm 3.8$ years & - & 2 & Inter-observer reliability $r=0.96$ \\
\hline Vasco [8] & 13 & 0 & $63.62(39-88)$ & - & - & 2 & Intra-rater reliability $k=1$ \\
\hline Tardella [9] & 34 & 6 & $56.4 \pm 13.42$ & $78 \pm 81.52$ months & $8.7 \pm 1.3$ & 2 & - \\
\hline
\end{tabular}

LUS lung ultrasound

43.16 (95\% CI 22.58-82.52), respectively. The AUC of LUS was 0.934 , and the $Q "$ index was 0.871 (Fig. 3), illustrating its great value for CTD-ILD diagnosis.

There were six different methods of LIS in the 11 articles, including 72, 65, 50, 14, 10, and all LIS (Table 4). The pooled sensitivity, specificity, PLR, NLR, DOR, and AUC of all LIS were 0.955 (95\% CI 0.873-0.991), 0.876 (95\% CI 0.798-0.932), 5.476 (95\% CI 2.597-11.55), 0.086 (95\% CI 0.035-0.211), 59.76 (95\% CI 21.05-1525.8), and 0.975 , respectively. The pooled sensitivity, specificity, PLR, NLR, and DOR of 14 LIS were 0.982 (95\% CI 0.904-1.000), 0.875 (95\% CI 0.710-0.965), 7.297 (95\% CI 3.050-17.45), 0.029 (95\% CI 0.006-0.147), and 292.30 (95\% CI 35.53-2403.8), respectively. All LIS and 14 LIS both had high sensitivity and specificity. In the search for a simplified and less time-consuming method, 14 LIS was found to be the best choice for LUS assessment in patients with CTD-ILD.

\section{Diagnostic value of LUS in different diseases}

There were six articles on SSc and three articles on RA (Table 5). The pooled sensitivity, specificity, PLR, NLR, and DOR of SSc were 0.839 (95\% CI 0.777-0.889), 0.870 (95\% CI 0.774-0.936), 6.203 (95\% CI 3.565-10.800), 0.191 (95\% CI 0.098-0.373), and 42.93 (95\% CI 17.75-103.79), respectively. The pooled sensitivity, specificity, PLR, NLR, and DOR of RA were 0.946 (95\% CI 0.818-0.993), 0.824 (95\% CI 0.730-0.896), 7.398 (95\% CI 0.768-74.220), 0.082 (95\% CI 0.024-0.275), and 80.24 (95\% CI 8.107-796.09), respectively. The AUC and the $Q^{*}$ index of SSc were 0.923 and 0.864 , respectively, while the AUC and $Q "$ index of RA were 0.981 and 0.939 , respectively (Fig. 3), indicating that LUS is a very useful tool for SSc and RA diagnosis.

\section{Discussion}

During the last decade, numerous studies on the role of LUS for ILD diagnosis in patients with CTD have been

Table 3 Study quality using the QUADAS tool

\begin{tabular}{|c|c|c|c|c|c|c|c|c|c|c|c|c|c|c|c|}
\hline First author [Ref] & 1 & 2 & 3 & 4 & 5 & 6 & 7 & 8 & 9 & 10 & 11 & 12 & 13 & 14 & Sum \\
\hline Delle [32] & $Y$ & $U$ & $\mathrm{Y}$ & $U$ & $\mathrm{Y}$ & $\mathrm{Y}$ & Y & $\mathrm{N}$ & $\mathrm{N}$ & $\mathrm{Y}$ & U & Y & $U$ & $U$ & 7 \\
\hline Tardella [10] & Y & Y & Y & Y & Y & Y & Y & Y & Y & Y & Y & Y & $U$ & Y & 13 \\
\hline Barskova [24] & Y & Y & Y & Y & Y & Y & Y & Y & Y & Y & U & Y & Y & Y & 13 \\
\hline Mohammadi [33] & Y & U & Y & $U$ & Y & Y & Y & Y & Y & Y & Y & Y & $U$ & Y & 11 \\
\hline Moazedi-Fuerst [34] & Y & Y & Y & $U$ & Y & Y & Y & Y & $\mathrm{N}$ & Y & U & Y & Y & Y & 11 \\
\hline Cogliati [7] & Y & Y & Y & $U$ & Y & Y & Y & Y & Y & Y & Y & Y & Y & Y & 13 \\
\hline Moazedi-Fuerst [23] & Y & $\mathrm{N}$ & Y & $U$ & Y & Y & Y & Y & $\mathrm{N}$ & Y & $U$ & Y & Y & Y & 10 \\
\hline Vizioli [35] & Y & Y & Y & Y & Y & Y & Y & Y & Y & Y & Y & Y & Y & Y & 14 \\
\hline Cakir [29] & Y & Y & Y & Y & Y & Y & Y & Y & Y & $U$ & U & Y & $\mathrm{N}$ & Y & 11 \\
\hline Vasco [8] & Y & Y & Y & Y & Y & Y & Y & Y & Y & Y & Y & Y & Y & Y & 14 \\
\hline Tardella [9] & Y & Y & Y & Y & Y & Y & Y & Y & Y & Y & Y & Y & Y & Y & 14 \\
\hline
\end{tabular}




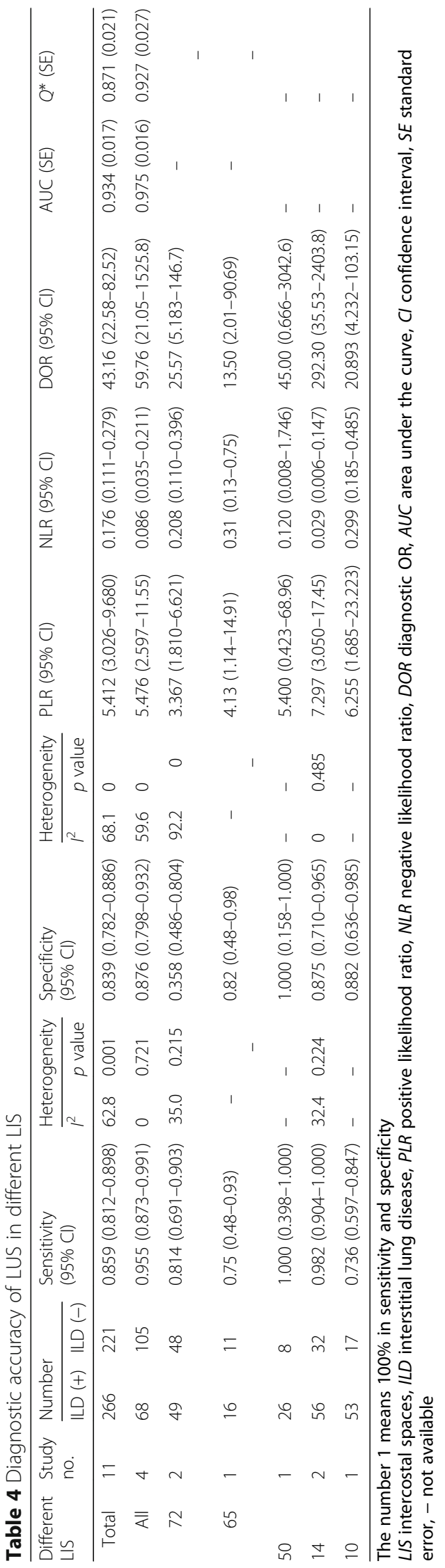




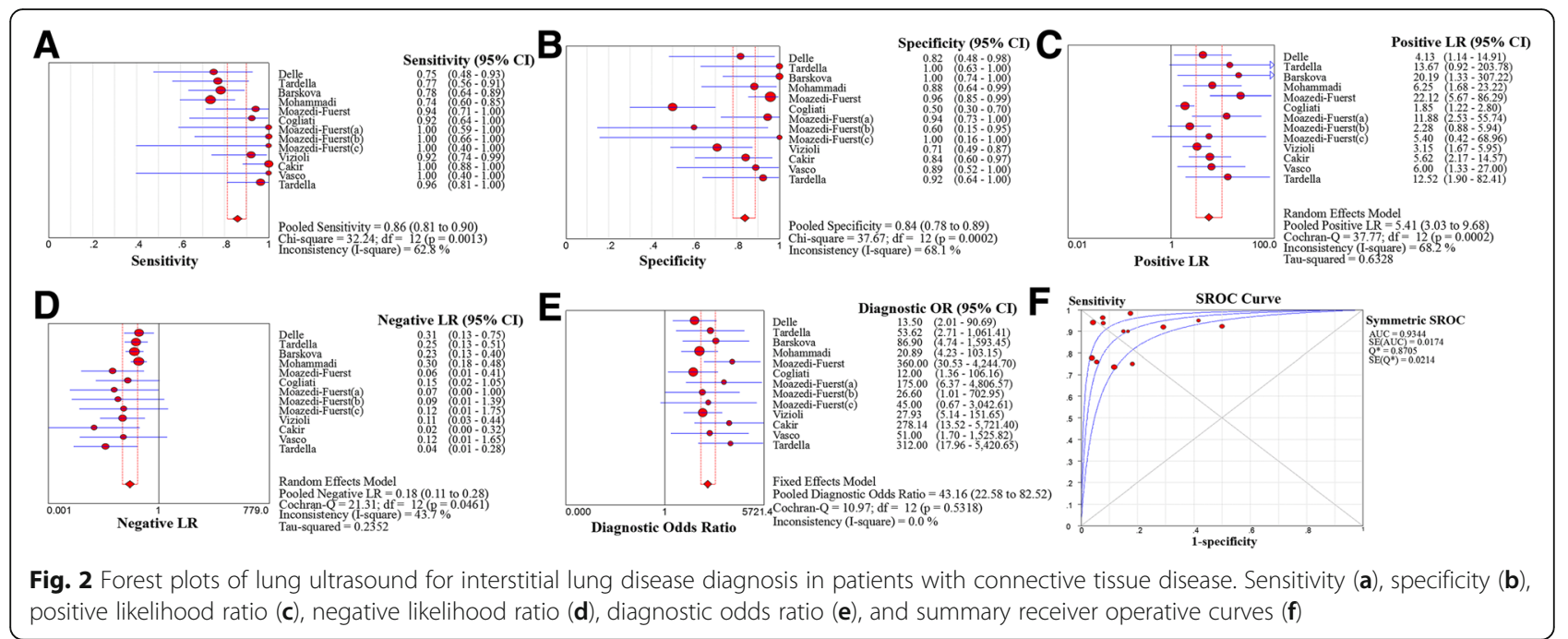

reported. Semi-quantitative data were measured by the sum of the number of B-lines, which were counted by the designed LIS [7, 9, 24]. However, there were extensive LUS scoring systems to assess the B-lines. Some studies referred to more LIS, such as all LIS, 72 LIS, and 50 LIS, which were time-consuming and difficult to extend $[7,10]$. Besides, without a uniform criteria method, it was hard to spread. Currently, there is no evidence about which LIS should be observed. Therefore, it is necessary to find a simplified and uniform method with fewer LIS for improved LUS diagnostic performance.

With the development of ultrasound technology, more and more studies have focused on ultrasound in lung diseases, which include pneumonia [25], neonatal respiratory distress syndrome [26], and interstitial lung disease [27]. In our meta-analysis, we concentrated on LUS in the diagnosis of ILD in patients with CTD. In a total of 487 patients with CTD, nearly half had the complication of ILD. The overall AUC was 0.934, indicating a high level of diagnostic performance. Our finding was similar to that in a recent study [28]. However, there were some differences between the two meta-analysis studies. First, in this meta-analysis, the emphasis was on finding a simplified LUS method, to facilitate daily clinical management, using the same uniform diagnostic criteria. Second, our meta-analysis included more studies than that of Song et al. Our meta-analysis included 11 studies with a total of 487 patients. The study by Song et al. included 6 studies with a total of 272 patients. Besides, more studies from the last 3 years were included

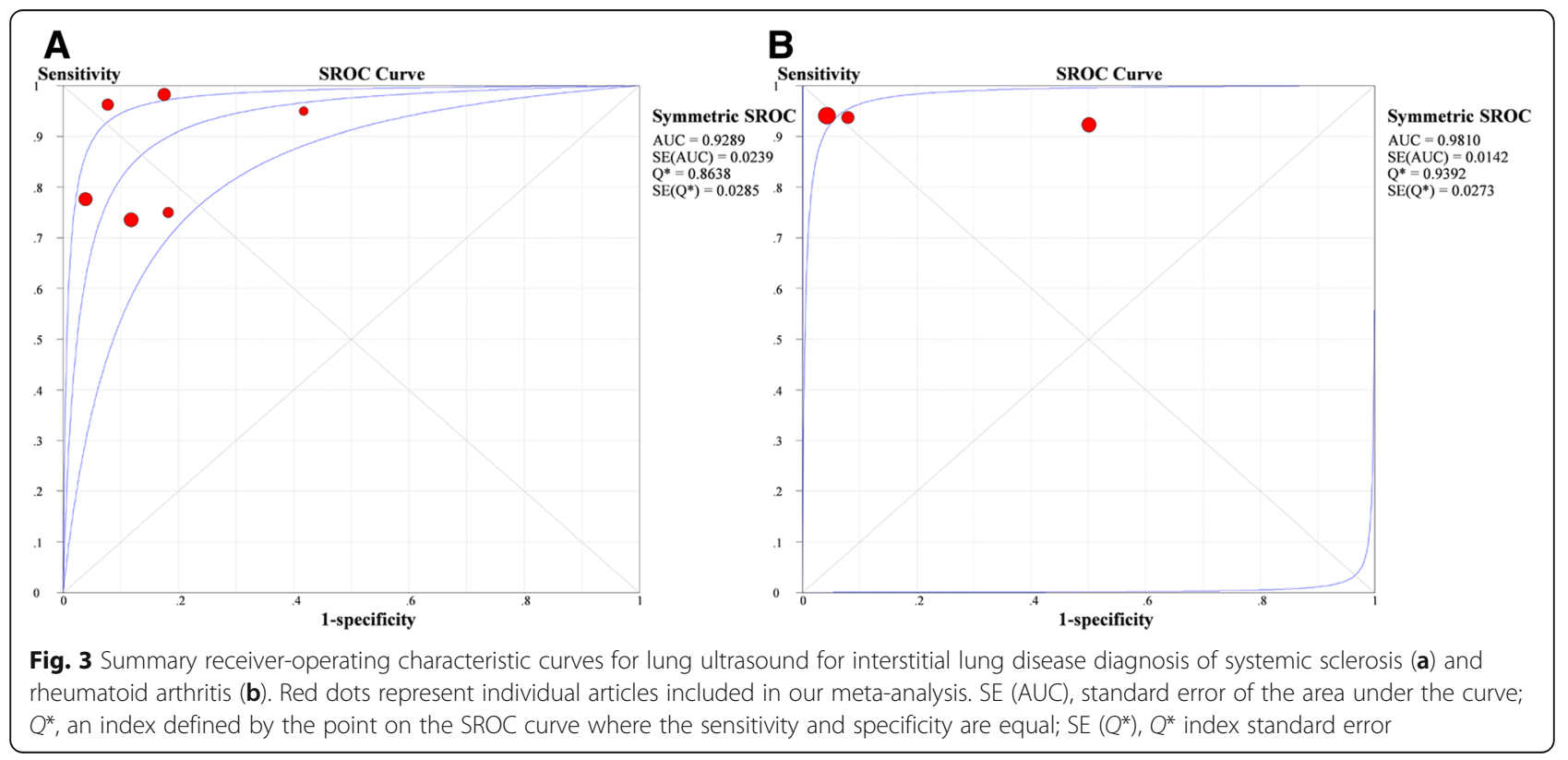




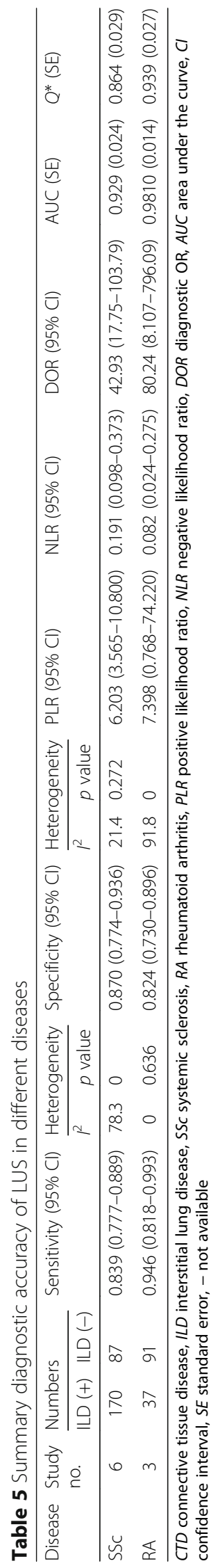


in our study. Third, our study included more different diseases in CTD, such as Sjögren's syndrome.

This meta-analysis found that all LIS and 14 LIS both had high diagnostic value. However, all LIS was time-consuming and not available for clinical practice. Therefore, 14 LIS may be the better choice for LUS diagnosis. 14 LIS consisted of bilateral, anterior, and posterior locations. For the unilateral chest, they selected 4 LIS including the second LIS on the parasternal lines and the fourth LIS on midclavicular, the anterior axillary, and the midaxillary lines respectively. For the posterior chest, they selected the eighth LIS on three lines, namely the paravertebral, the subscapular, and the posterior axillary lines. Among the reasons for choosing these LIS were the demonstrated higher prevalence rate of B-lines in these fields and the ease of evaluation by LUS $[14,29]$. To the best of our knowledge, there is no meta-analysis about which LIS should be evaluated in a modified LUS for diagnosis of CTD-ILD. We found that the overall diagnostic performance of 14 LIS was higher than that of 72 LIS. The possible reasons may be associated with different diagnostic criteria, disease duration, deviations among patients, etc.

We also performed subgroup analysis by diseases. Since ILD is common in patients with SSc [30] and RA [31], most of the studies were focused on these two diseases. In our study, the results were not significantly different between them, showing that LUS was of a great diagnostic value for both diseases. In comparison, the diagnostic efficiency of LUS in RA was slightly higher than SSc. The DOR and AUC of RA were 80.24(95\% CI 8.107-796.09) and 0.981, respectively. The DOR and AUC of SSc were $42.93(95 \%$ CI $17.75-103.79)$ and 0.929 , respectively. The difference was possibly related to the different observed LIS, probe types, probe frequency, disease duration, etc. The number of LIS observed in patients with RA was all and 72 LIS, but in patients with SSc was diverse, including all, 72, 65, 14, and 10 LIS. The number of studies in SSc was more than that in RA. Moreover, the number of patients with SSc was larger than those with RA. Above all, the overall accuracy was relatively lower in SSc, but it still demonstrated that LUS was a useful method for the diagnosis of ILD in patients with SSc.

There was heterogeneity in this study. For the diagnostic accuracy in different LIS, the $I^{2}$ of all LIS and 72 LIS were 59.6 and 92.2 in specificity, respectively. The reasons may be connected with different diseases, territory, and basic characteristic information. For the summary diagnostic accuracy in all studies, there was also heterogeneity in sensitivity and specificity. It is possible that it was related to demographic characteristics, such as probe types, probe frequency, number of LIS, disease duration, and reproducibility between operators.
Our meta-analysis had some limitations. First, the search strategy was restricted to studies in the English language. So, certain studies were missing. Second, there was no uniform standard set of criteria for ILD diagnosis by LUS. The result of sensitivity and specificity showed considerable variation. The sensitivity of our study was from 73.6 to $100 \%$, and specificity was from 50 to $100 \%$. The pooled data produced a more reliable result and decreased the variation. The study quality was evaluated using the tool of QUADAS. The quality of some studies was low, which would have an impact on our results. Third, there was heterogeneity among the articles, but we performed subgroup analysis, to find the possible reasons. Fourth, the number of studies was small; thus, there was not enough data to prove the diagnostic accuracy.

\section{Conclusions}

We found a modified and simplified method of LUS, by scanning 14 LIS in a short time, which had a very high sensitivity and specificity. This LUS method may be a good choice for the assessment of ILD in patients with CTD. However, our data had some limitations, and more evidence is needed.

\section{Abbreviations \\ AUC: Area under the curve; $\mathrm{Cl}$ : Confidence interval; CTD: Connective tissue disease; DOR: Diagnostic OR; HRCT: High-resolution computed tomography; ILD: Interstitial lung disease; LIS: Intercostal spaces; LUS: Lung ultrasound; MSH: Medical Subject Headings; NLR: Negative likelihood ratio; PLR: Positive likelihood ratio; QUADAS: Quality Assessment of Diagnostic Accuracy Studies; RA: Rheumatoid arthritis; SE: Standard error; SLE: Systemic lupus erythematosus; SROC: Summary receiver-operating characteristic curves; SS: Sjögren's syndrome; SSc: Systemic sclerosis}

\section{Acknowledgements}

Not applicable.

\section{Funding}

The present study was supported by the Science Technology and Innovation Commission of Shenzhen Municipality in China (grant no.

JCYJ20170413100222613), Natural Science Foundation of Guangdong Province (grant no.2018A0303130228) and the Health and Family Planning

Commission of Shenzhen Municipality in China (grant no. SZSM201512026).

\section{Availability of data and materials} Not applicable.

Authors' contributions

$\mathrm{ZH}$ and $\mathrm{LL}$ conceived and designed the study. $\mathrm{HQ}, \mathrm{WW}$, and $\mathrm{XM}$ retrieved the studies and extracted data. DS and SF analyzed the data. $\mathrm{HQ}$ and $\mathrm{ZH}$ wrote the paper. All the authors have seen and approved the manuscript.

Ethics approval and consent to participate

Not applicable.

Consent for publication

Not applicable.

Competing interests

The authors declare that they have no competing interests. 


\section{Publisher's Note}

Springer Nature remains neutral with regard to jurisdictional claims in published maps and institutional affiliations.

Received: 31 December 2018 Accepted: 2 April 2019

Published online: 11 April 2019

\section{References}

1. Khanna D, Mittoo S, Aggarwal R, Proudman SM, Dalbeth N, Matteson EL, Brown K, Flaherty K, Wells AU, Seibold JR, et al. Connective tissue diseaseassociated interstitial lung diseases (CTD-ILD) - report from OMERACT CTDILD working group. J Rheumatol. 2015;42(11):2168-71.

2. Vij R, Strek ME. Diagnosis and treatment of connective tissue diseaseassociated interstitial lung disease. Chest. 2013;143(3):814-24.

3. Desai SR, Veeraraghavan S, Hansell DM, Nikolakopolou A, Goh NS, Nicholson AG, Colby TV, Denton CP, Black CM, du Bois RM, et al. CT features of lung disease in patients with systemic sclerosis: comparison with idiopathic pulmonary fibrosis and nonspecific interstitial pneumonia. Radiology. 2004 232(2):560-7.

4. Devaraj A, Wells AU, Hansell DM. Computed tomographic imaging in connective tissue diseases. Semin Respir Crit Care Med. 2007;28(4):389-97.

5. Ohno Y, Koyama H, Yoshikawa T, Seki S. State-of-the-art imaging of the lung for connective tissue disease (CTD). Curr Rheumatol Rep. 2015;17(12):69.

6. Volpicelli G, Elbarbary M, Blaivas M, Lichtenstein DA, Mathis G, Kirkpatrick AW, Melniker L, Gargani L, Noble VE, Via G, et al. International evidencebased recommendations for point-of-care lung ultrasound. Intensive Care Med. 2012;38(4):577-91.

7. Cogliati C, Antivalle M, Torzillo D, Birocchi S, Norsa A, Bianco R, Costantino G, Ditto MC, Battellino M, Puttini PCS, et al. Standard and pocket-size lung ultrasound devices can detect interstitial lung disease in rheumatoid arthritis patients. Rheumatol. 2014;53(8):1497-503.

8. Vasco PG, de Luna Cardenal G, Garrido IM, Pinilla JM, Rodriguez GF, Mateo J, Ruiz DC. Assessment of interstitial lung disease in Sjogren's syndrome by lung ultrasound: a pilot study of correlation with high-resolution chest tomography. Intern Emerg Med. 2017:12(3):327-31.

9. Tardella M, Di Carlo M, Carotti M, Filippucci E, Grassi W, Salaffi F. Ultrasound B-lines in the evaluation of interstitial lung disease in patients with systemic sclerosis: cut-off point definition for the presence of significant pulmonary fibrosis. Medicine. 2018:97(18):e0566.

10. Tardella M, Gutierrez M, Salaffi F, Carotti M, Ariani A, Bertolazzi C, Filippucci E, Grassi W. Ultrasound in the assessment of pulmonary fibrosis in connective tissue disorders: correlation with high-resolution computed tomography. J Rheumatol. 2012;39(8):1641-7.

11. Whiting P, Rutjes AW, Reitsma JB, Bossuyt PM, Kleijnen J. The development of QUADAS: a tool for the quality assessment of studies of diagnostic accuracy included in systematic reviews. BMC Med Res Methodol. 2003;3:25.

12. Higgins JP, Thompson SG. Quantifying heterogeneity in a meta-analysis. Stat Med. 2002;21(11):1539-58

13. Zamora J, Abraira V, Muriel A, Khan K, Coomarasamy A. Meta-DiSc: a software for meta-analysis of test accuracy data. BMC Med Res Methodol. 2006:6:31

14. Gutierrez M, Salaffi F, Carotti M, Tardella M, Pineda C, Bertolazzi C, Bichisecchi E, Filippucci E, Grassi W. Utility of a simplified ultrasound assessment to assess interstitial pulmonary fibrosis in connective tissue disorders - preliminary results. Arthritis Res Ther. 2011;13(4):R134.

15. Vassalou EE, Raissaki M, Magkanas E, Antoniou KM, Karantanas AH. Lung ultrasonography in patients with idiopathic pulmonary fibrosis evaluation of a simplified protocol with high-resolution computed tomographic correlation. J Ultrasound Med. 2018;37(3):689-96.

16. Vassalou EE, Raissaki M, Magkanas E, Antoniou KM, Karantanas AH. Modified lung ultrasonographic technique for evaluation of idiopathic pulmonary fibrosis: lateral decubitus position. J Ultrasound Med. 2017;36(12):2525-32.

17. Gargani L, Doveri M, D'Errico L, Frassi F, Bazzichi ML, Delle Sedie A, Scali MC, Monti S, Mondillo S, Bombardieri S, et al. Ultrasound lung comets in systemic sclerosis: a chest sonography hallmark of pulmonary interstitial fibrosis. Rheumatology (Oxford). 2009;48(11):1382-7.

18. Fernandez IP, Nunez EP, Selva-O'Callaghan A, Fierro EC, Martinez-Gomez X, Vilardell-Tarres M. Correlation of ultrasound B-lines with high-resolution computed tomography in antisynthetase syndrome. Clin Exp Rheumatol. 2014;32(3):404-7.
19. Gigante A, Fanelli FR, Lucci S, Barilaro G, Quarta S, Barbano B, Giovannetti A, Amoroso A, Rosato E. Lung ultrasound in systemic sclerosis: correlation with high-resolution computed tomography, pulmonary function tests and clinical variables of disease. Intern Emerg Med. 2016;11(2):213-7.

20. Pinal-Fernandez I, Pallisa-Nunez E, Selva-O'Callaghan A, Castella-Fierro E, Simeon-Aznar CP, Fonollosa-Pla V, Vilardell-Tarres M. Pleural irregularity, a new ultrasound sign for the study of interstitial lung disease in systemic sclerosis and antisynthetase syndrome. Clin Exp Rheumatol. 2015;33(4):S136-41.

21. Moazedi-Fuerst FC, Zechner PM, Tripolt NJ, Kielhauser SM, Brickmann K, Scheidl S, Lutfi A, Graninger WG. Pulmonary echography in systemic sclerosis. Clin Rheumatol. 2012;31(11):1621-5.

22. Buda N, Piskunowicz M, Porzezinska M, Kosiak W, Zdrojewski Z. Lung ultrasonography in the evaluation of interstitial lung disease in systemic connective tissue diseases: criteria and severity of pulmonary fibrosis analysis of 52 patients. Ultraschall Med. 2016;37(4):375-81.

23. Moazedi-Fuerst F, Kielhauser S, Brickmann K, Tripolt N, Meilinger M, Lutfi A, Graninger W. Sonographic assessment of interstitial lung disease in patients with rheumatoid arthritis, systemic sclerosis and systemic lupus erythematosus. Clin Exp Rheumatol. 2015;33(4):S87-91.

24. Barskova T, Gargani L, Guiducci S, Randone SB, Bruni C, Carnesecchi G, Conforti ML, Porta F, Pignone A, Caramella D, et al. Lung ultrasound for the screening of interstitial lung disease in very early systemic sclerosis. Ann Rheum Dis. 2013:72(3):390-5.

25. Pereda MA, Chavez MA, Hooper-Miele CC, Gilman RH, Steinhoff MC, Ellington LE, Gross M, Price C, Tielsch JM, Checkley W. Lung ultrasound for the diagnosis of pneumonia in children: a meta-analysis. Pediatrics. 2015; 135(4):714-22

26. Hiles M, Culpan AM, Watts C, Munyombwe T, Wolstenhulme S. Neonatal respiratory distress syndrome: chest X-ray or lung ultrasound? A systematic review. Ultrasound. 2017:25(2):80-91.

27. Wang YK, Gargani L, Barskova T, Furst DE, Cerinic MM. Usefulness of lung ultrasound B-lines in connective tissue disease-associated interstitial lung disease: a literature review. Arthritis Res Ther. 2017;19(1):206.

28. Song GG, Bae SC, Lee YH. Diagnostic accuracy of lung ultrasound for interstitial lung disease in patients with connective tissue diseases: a metaanalysis. Clin Exp Rheumatol. 2016;34(1):11-6.

29. Cakir Edis E, Hatipoglu ON, Pamuk ON, Mutlucan Eraslan R, Aktoz M, Tuncel SA. Effectiveness of thoracic ultrasonography in the evaluation of the severity of pulmonary involvement in patients with systemic sclerosis. Archives of Rheumatol. 2016;31(4):364-70.

30. Wells AU, Steen $\mathrm{V}$, Valentini G. Pulmonary complications: one of the most challenging complications of systemic sclerosis. Rheumatology (Oxford) 2009:48(Suppl 3):iii40-4.

31. Young A, Koduri G. Extra-articular manifestations and complications of rheumatoid arthritis. Best Pract Res Clin Rheumatol. 2007;21(5):907-27.

32. Delle Sedie A, Doveri M, Frassi F, Gargani L, D'Errico G, Pepe P, Bazzichi L, Riente L, Caramella D, Bombardieri S. Ultrasound lung comets in systemic sclerosis: a useful tool to detect lung interstitial fibrosis. Clin Exp Rheumatol. 2010;28(5 Suppl 62):S54.

33. Mohammadi A, Oshnoei S, Ghasemi-rad M. Comparison of a new, modified lung ultrasonography technique with high-resolution $C T$ in the diagnosis of the alveolo-interstitial syndrome of systemic scleroderma. Med Ultrason. 2014;16(1):27-31.

34. Moazedi-Fuerst FC, Kielhauser SM, Scheidl S, Tripolt NJ, Lutfi A, Yazdani-Biuki B, Dejaco C, Graninger WB. Ultrasound screening for interstitial lung disease in rheumatoid arthritis. Clin Exp Rheumatol. 2014:32(2):199-203.

35. Vizioli L, Ciccarese F, Forti P, Chiesa AM, Giovagnoli M, Mughetti M, Zompatori M, Zoli M. Integrated use of lung ultrasound and chest X-ray in the detection of interstitial lung disease. Respiration. 2017;93(1):15-22. 